\title{
DESIGN OF A HAPTIC DEVICE FOR DISMANTLING PROCESSES USING EXCAVATOR
}

\author{
Dongnam Kim, Kyeongwon Oh \\ Korea Univ. Graduate School \\ 5Ga-1, Anam-Dong \\ Sungbuk-Gu, Seoul, Korea \\ \{smileast, locoski \}@korea.ac.kr
}

\author{
Daehie Hong \\ Korea University, Dept of ME \\ 5Ga-1, Anam-Dong, \\ Sungbuk-Gu, Seoul, Korea \\ dhhong@korea.ac.kr
}

\author{
Jong-Hyup Park and Suk-Hie Hong \\ Naekyung Engineeing Co., Ltd. \\ 5-1 Sewon building, Yangjae-Dong \\ Seocho-Gu, Seoul, Korea \\ $\{$ nk2900, hongcon\}@korea.com
}

\begin{abstract}
Since the processes of dismantling are very dangerous, there have been many studies to develop remote operating devices using joystick. In this paper, in order to improve the operability of the dismantling equipment that is usually an excavator, a novel concept of tele-operated haptic device is proposed. Operators who use this haptic device with additional environmental sensing devices can work safely away from the dangerous sites. First, based on the concept design of the haptic device, its workspace is analyzed and the workspace mapping from the haptic device to the excavator is explored. Second, after 7 steps of the excavating processes are defined, the kinematics which deals with the conversion from the 3 dimensional position information of the haptic device to the joint variable information of the backhoe is included in this paper. Lastly, 3D graphical simulation of both haptic device and the backhoe will be shown. This new design of the haptic device can be easily manufactured and gives the workers very convenient and transparent remote control capability.
\end{abstract}

\section{KEYWORDS}

Haptic Device, Excavator, Inverse Kinematics, Dismantling Process

\section{INTRODUCTION}

Nowadays, there are many construction sites where new skyscrapers are built. Also, remodeling or dismantling of existing buildings is unavoidable due to the limitation of land. Some methods and/or equipments have been developed for the dismantling processes but they can not sometimes be used due to the size of building and environmental factor. From this reason, mechanical crushing process is still very common method in dismantling sites. Furthermore, equipments that are used in construction sites are usually adapted to dismantle the building. Especially, an excavator equipped with crusher at its end is a good example of the dismantling equipments. However, the dismantling process is very dangerous if operator rides on the excavator at first hand. So, tele-operation system to manage the excavator from remote site is inevitable to assure the operator's safety.

There have been some recent studies related to the tele-operation of the excavator. First, kinematics analysis and simulation of construction devices were conducted by 
Frimpong ${ }^{[1]}$. Furthermore, Frankel ${ }^{[2]}$ studied remote control of excavator with commercial haptic device, Phantom. Finally, haptic device of excavator which was operated in underwater was studied by Hirabayashi ${ }^{[3]}$.

The remote controller, that is, haptic device of excavator for dismantling processes, is the main theme of this paper. The haptic device is designed based on the kinematics of the excavator, which can cover 3-dimensional workspace. Moreover, when the excavator is remotely controlled, the forces exerted to the links and joints of the excavator can be very high but not transferred to the operator, which may result in the damage of the excavator in the extreme case. In order to solve this problem, the remote controller is designed to feedback the force to the operator. In this paper, kinematics analyses of the designed haptic device as well as the excavator are also studied. Based on these analyses, the transformation between the haptic device and the excavator is defined. Finally, 3 dimensional modeling of the excavator and the haptic device by OpenGL program are shown. The models are applied to digging operation through computer simulations.

\section{KINEMATICS OF EXCAVATOR}

\subsection{Kinematics of Excavator for Haptic}

There are so many kinds of backhoes in the world but excavator whose joints are represented by 4 motion, swing, boom, arm, and bucket, is chosen in this paper. This excavator can be easily seen in dismantling sites. Based on this motion, Fig. 1 shows that 4 coordinates of each links of the excavator. Swing joint axis of excavator is represented by $Z_{e 1}$, and the remaining three joint axes, boom, arm, bucket are also represented.

After the modeling of excavator, forward and inverse kinematics analysis is also studied. When the haptic devices are designed, the inverse kinematics analysis is more important. Because the operator wants to control the bucket link, the pose of bucket link is determined by the haptic device. So, according to the bucket's pose, four joint angles of excavator are controlled by the inverse kinematics analysis.

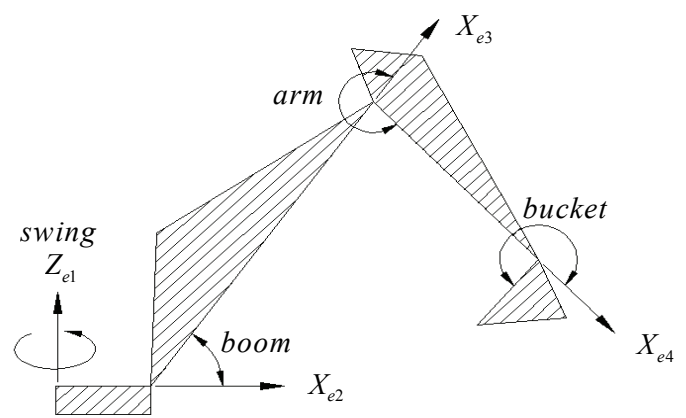

Figure 1 Kinematics Modeling of Excavator

Excavator's degree of freedom is 4 , at the same time, bucket can move in 3-dimensional space. So, the bucket movement can be represented with haptic stylus in 3D space. However, when excavator's motion for real digging operations is examined, it is observed that the swing motion is nearly not used. That is, the excavator's main motion is controlled by 3 DOF without swing, which determines the bucket's 2D motion. The swing motion is usually used to move the dug earth to other side. If the swing motion is separated from the digging motion and can be controlled by the haptic device's another reserved joint, operator can control the excavator more easily.

Therefore, inverse kinematics analysis of excavator which is 2-dimensional kinematics problem to control the boom, arm, and bucket will be shown in the next section.

\subsection{Kinematics Analysis of Excavator}

When the operator is using the haptic device, the angle and position of bucket can be obtained by the absolute position of the haptic stylus. In fact, the angle of bucket and the position of bucket tip are important to control the excavator. However, if the position of a rotational axis of bucket $\left(Z_{e 4}\right)$ and its angle can be controlled separately from the bucket tip's position and angle, more intuitive controllability can be obtained. So, important point to control the excavator is distal joint position of arm, and this information is used in inverse kinematics analysis. Following equations are inverse kinematics equations to calculate the angles of boom and $\operatorname{arm}^{[2]}$. 


$$
\begin{gathered}
\theta_{e 2}=\tan ^{-1}\left(\frac{r_{24}(y)}{r_{24}(x)}\right)+\cos ^{-1}\left(\frac{a_{2}{ }^{2}+r_{24}{ }^{2}-a_{3}^{2}}{2 a_{2} r_{24}}\right) \\
\theta_{e 3}=\pi+\cos ^{-1}\left(\frac{a_{2}{ }^{2}+a_{3}{ }^{2}-r_{13}{ }^{2}}{2 a_{2} a_{3}}\right)
\end{gathered}
$$

$a_{2}$ : Boom length (distance from $Z_{e 2}$ to $Z_{e 3}$ )

$a_{3}$ : Arm length (distance from $Z_{e 3}$ to $Z_{e 4}$ )

$r_{24}$ : Distance from $Z_{e 2}$ to $Z_{e 4}$

$\theta_{e i}:$ Angle or each link $(i=1,2,3,4)$

The angle control of bucket is also designed with separate joint from boom and arm control in the haptic device like the swing control in the previous section. Due to simple control method, the bucket is not included in the kinematic analysis.

\subsection{Workspace Analysis of Excavator}

Fig. 2 shows the workspace of excavator from side view. First, the dual line represents the boom link of excavator whose length is $2 \mathrm{~m}$, and the thick line does the arm link whose length is $1.4 \mathrm{~m}$. The cross dots represents the trajectory points of distal joint of the boom when the boom joint moves from $(-30)$ to $(60)$ degree. In the figure, the rotational axis of boom is on the point $(1 \mathrm{~m}$, $1 \mathrm{~m})$. And the range of arm angle is from (240) to (360) degree. So, the black little dots represent the area of distal joint of the arm. That is, the rotational axis of the bucket is within this area. In consequence, this area defines the workspace of the excavator.

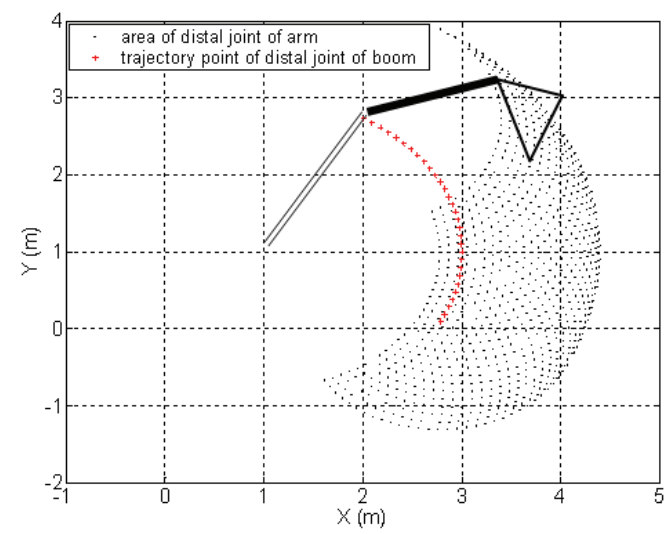

Figure 2 Workspace of Excavator (Joint Position of Arm Link)

\section{DESIGN OF HAPTIC DEVICE}

\subsection{Design Concept of Haptic Device}

In this paper, a haptic device is designed, such that operator can work with intuitive bucket control. So, novice operator can use the excavator easily unlike the conventional controller. Fig. 3 shows the conceptul picture of the haptic device. Coordinate assignment is also shown in the figure with Denavit-Hartenberg coordinate system and the used parameter values are defined in Table 1. The degree of freedom is 4 and swing and bucket control is directly connected to two joints of haptic device. For example, $\theta_{h 1}$ controls the swing motion and $\theta_{h 4}$ does the bucket motion directly.

The position of arm tip is controlled by $\theta_{h 2}$ and $d_{h 3}$. Therefore, the angles of boom and arm $\left(\theta_{e 2}, \theta_{e 3}\right)$ are calculated by inverse kinematics analysis.

Table 1 DH-parameter of haptic device

\begin{tabular}{c|c|c|c|c}
\hline \hline$i$ & $\theta_{h i}$ & $\alpha_{h i}$ & $a_{h i}$ & $d_{h i}$ \\
\hline \hline 2 & $\pi / 2+\theta_{h 2}$ & $-\pi / 2$ & 0 & 0 \\
\hline 3 & 0 & 0 & 0 & $d_{h 3}$ \\
\hline
\end{tabular}

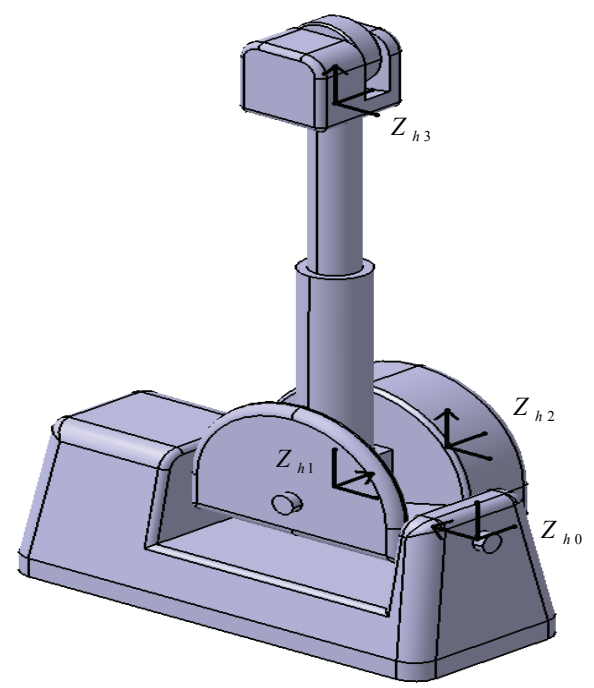

Figure 3 Conceptual design of Haptic Device with D-H Coordinates (only $Z$ axes are shown) 


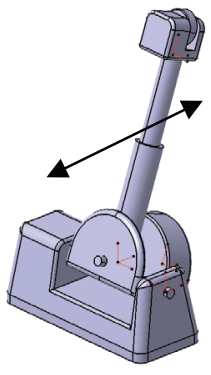

(a) $\theta_{h 1}$

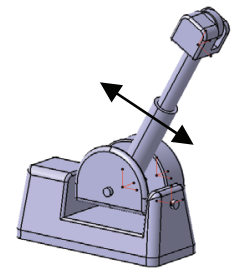

(b) $\theta_{h 2}$

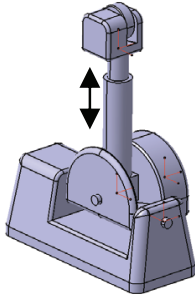

(c) $d_{h 3}$
Figure 4 Motion of Haptic Device

Fig. 4 shows how to move the joints of haptic device. Fig. 4 (a) is a picture of swing motion control. Fig. 4 (b), (c) show the control of $\theta_{h 2}$ and $d_{h 3}$ which corresponds to the bucket joint position. This means that operator can move the haptic stylus in 2-D plane and these information are transferred to the excavator to control the $\theta_{e 2}$ and $\theta_{e 3}$, the angles of boom and arm, respectively. Lastly, the force measured at the bucket joint is usually very high, because the bucket is responsible for main operation of excavation or dismantling. Therefore, it is more advantageous for the operator to control the bucket angle by a separate joint like a thumbwheel which is comfortable to operate. This thumbwheel which directly controls $\theta_{h 4}$ is set up on the haptic stylus.

Fig. 5 shows internal mechanism of the haptic device. There are 4 actuators, electrical motors, and also 4 encoders. The encoders read the angle of each link, and the actuators are used to feedback the forces from the excavator to operator.

\subsection{Kinematics Analysis of Haptic Device}

To calculate the position vector of the end point of the haptic stylus relative to base frame, transformation matrix $T$ is used like following equation.

$$
{ }^{i-1} T_{i}=\left[\begin{array}{cccc}
\cos \theta_{i} & -\cos \alpha_{i} \sin \theta_{i} & \sin \alpha_{i} \sin \theta_{i} & a_{i} \cos \theta_{i} \\
\sin \theta_{i} & \cos \alpha_{i} \cos \theta_{i} & -\sin \alpha_{i} \cos \theta_{i} & a_{i} \sin \theta_{i} \\
0 & \sin \alpha_{i} & \cos \alpha_{i} & d_{i} \\
0 & 0 & 0 & 1
\end{array}\right] \text { (3) }
$$

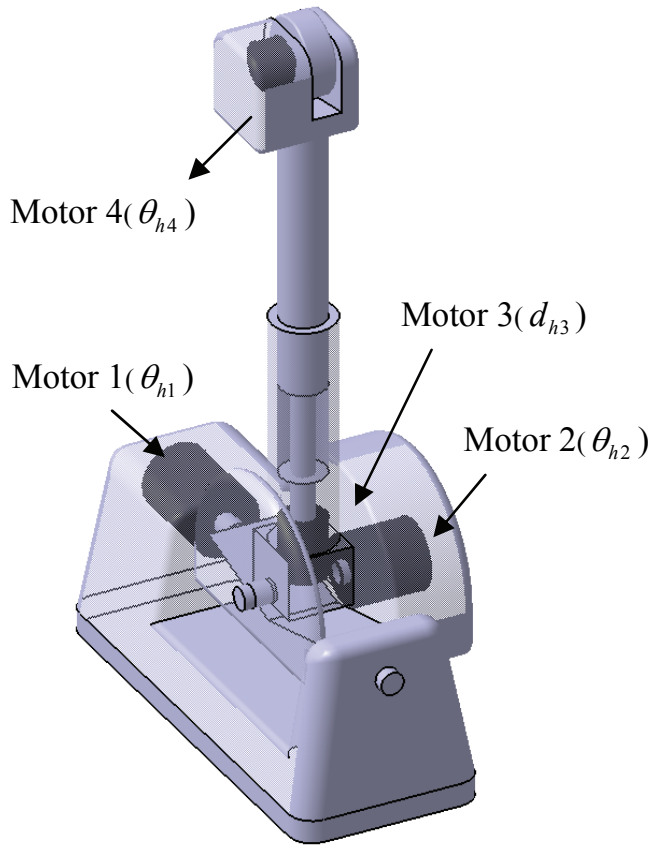

Figure 5 Force Feedback Mechanism of 4 DOF Haptic Device Using Electrical Motors

When the drive mapping between the haptic device and the excavator is defined, $\theta_{h 2}$ and $d_{h 3}$ are control factor to find out the position of bucket rotational axis. So, from the base frame of the haptic to the haptic stylus, transformation matrix is obtained by multiplication of two matrix, ${ }^{1} T_{2}$ and ${ }^{2} T_{3}$. DH parameters are already known and these values are the components of the transformation matrix. For forward kinematics of the haptic device, $\theta_{h 2}$ and $d_{h 3}$ are given, and the stylus position should be calculated. It is solved by following equation.

$$
{ }^{1} P={ }^{1} T_{2}{ }^{2} T_{3}^{3} P
$$

${ }^{1} P$ is the position vector which represents the stylus position relative to the coordinate $1,{ }^{3} P$ is also the position vector relative to the coordinate 3 . The stylus position is coincident with the origin of the coordinate 3 , that is, ${ }^{3} P$ is $(0,0,0,1)^{T}$. In summary, by using the haptic device, operator can control the whole excavator joints. 


\subsection{Workspace Analysis of Haptic Device}

Fig. 6 is the picture of workspace of haptic device, and this is shown from side view of haptic device. That is, the workspace is calculated from the two parameters, $\theta_{h 2}$ and $d_{h 3}$. The range of $\theta_{h 2}$ is from (-50) degree to (50) degree and the range of $d_{h 3}$ is from (0) $\mathrm{m}$ to $(0.3) \mathrm{m}$. The rotational axis of $\theta_{h 2}$ is on the point $(0 \mathrm{~m}, 0 \mathrm{~m})$. Workspace analysis is a initial step to control the excavator with haptic device.

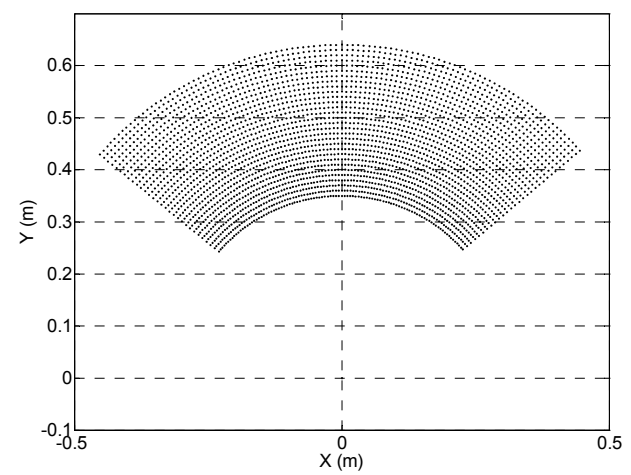

Figure 6 Workspace of Haptic Device

\section{MAPPING FROM HAPTIC DEVICE TO EXCAVATOR}

Pose information from the haptic stylus position and the angle of thumbwheel controlled by operator decides the angles of excavator joints. In the previous section, the excavator swing motion is controlled by $\theta_{h 1}$. If $\theta_{h 1}$ is minus value, excavator swings left. It turns right otherwise. Next, $\theta_{e 2}$ and $\theta_{e 3}$ are controlled by $\theta_{h 2}$ and $d_{h 3}$. First, the haptic stylus position is read by forward kinematics and these values are converted to the position of bucket joints. This position can be obtained by scaling the workspace between the haptic device and the excavator. Fig. 7 shows the work space mapping from the haptic device to the excavator. In sections 2.3 and 3.3, each workspace is already obtained. Scaling factor 17.5 is used to enlarge the small workspace of the haptic device, and entire workspace is translated on Y-axis by (-7). In the results, the workspace of the haptic device covers that of excavator, so that the haptic device can control the excavator completely. Lastly, the thumbwheel value, $\theta_{h 4}$, is directly connected to the bucket angle. This mapping ratio is $1: 1$, in this paper. However, operator can finely control the bucket by changing this ratio.

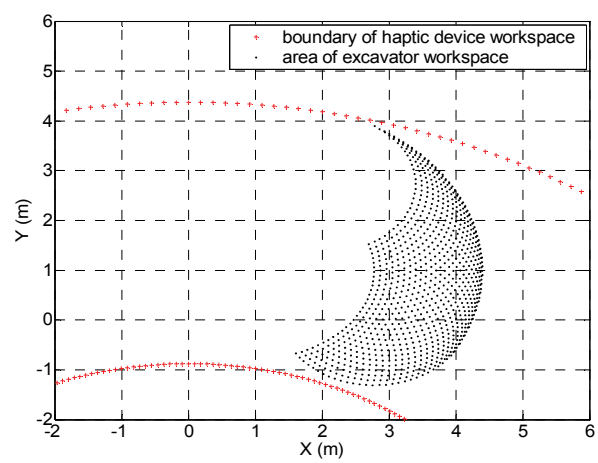

Figure 7 Workspace Mapping From Haptic Device to Excavator

\section{SIMULATION RESULTS}

In the previous sections, kinematics and workspace analysis of excavator and haptic device are derived. From now, simulation of haptic drive will be shown. Fig. 8 shows the models of the excavator and the haptic device with OpenGL program. OpenGL modeling of haptic device is simple to check the motion of each link. Simulation is applied to digging process and this is composed of 7 sequences like followings.

1. Reach out with the bucket to soil

2. Plunge down into the soil

3. Draw the bucket to fill it with dirt

4. Lift the bucket

5. Swing the bucket over to the spoil pile

6. Unload the bucket onto the spoil pile

7. Swing the bucket back and repeat

Table 2 is the pictures of digging processes in order. In each process, the movement of excavator and haptic device is shown.

This program works in real time. Mouse is used to control the haptic in the computer simulation. $2 \mathrm{D}$ position of the mouse represents the $2 \mathrm{D}$ position of bucket joint, and thumbwheel is directly used to control the bucket like haptic device. And swing control is carried out by 
pushing the arrow keys of keyboard. In Fig. 8, a thumbwheel of haptic is represented like $3^{\text {rd }}$ link instead of one circle like thumbwheel to check the movability. And swing motion controller is cut out, because this joint is not parallel with the other joints.

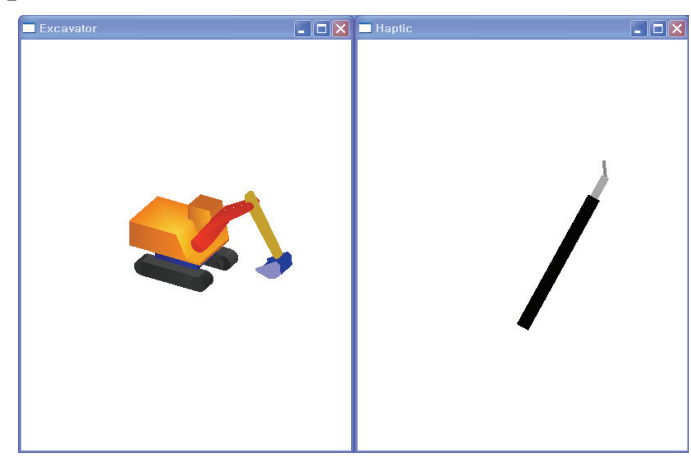

Fig. 8 OpenGL Modeling of Excavator and Haptic

\section{CONCLUSION}

In this paper, new haptic device was designed to control the excavator. Kinematics and workspace analysis were conducted and OpenGL graphical simulation of the haptic device was also included. As a result, the designed haptic device was proven to be very intuitive and convenient.

\section{ACKNOWLEDGMENT}

This research was supported by a grant(code:06 Construction Core B04) from Construction Core Technology Program funded by Ministry of Construction \& Transportation of Korean government and BK21.

\section{REFERENCES}

[1] S. Frimpong and Y. LI, 2005, Virtual prototype simulation of hydraulic shovel kinematics for spatial characterization in surface mining operations, Int. J. of Surface Mining, Reclamation and Environment, Vol. 19, No. 4, pp. 238 250.

[2] J. G. Frankel, 2004, Development of a Haptic Backhoe Testbed, A Thesis of Master of Science, School of Mechanical Engineering, Georgia Institute of Technology.
[3] T. Hirabayashi and T. Yamamoto, 2006, Experiment of Teleoperation of Underwater Backhoe With Haptic Information, Proceedings $23^{\text {rd }}$ Int. Sym. on Automation and Robotics in Construction, pp. 36 41.

Table 2 Simulation (Digging Processes)

\begin{tabular}{r|r|r}
\hline \hline Process 1 & Excavator & Haptic \\
\hline Process 1 & & \\
\hline Process 2 & & \\
\hline Process 3 & & \\
\hline Process 5 & & \\
\hline
\end{tabular}

